\title{
Rechtsextremismus als Herausforderung vor Ort
}

\section{Die Kommunen müssen handeln und brauchen dafür Unterstützung}

\author{
Uwe Lübking
}

Uwe Lübking ist Beigeordneter für Recht, Soziales und Gesundheitspolitik des Deutschen Städte- und Gemeindebundes und Mitglied im Beirat der Blätter der Wohlfahrtspflege. Internet http://www.dstgb.de
Rechtsextremistische Organisationen nutzen die in Teilen der Bevölkerung vorhandene Staatsverdrossenheit aus und versuchen, gesellschaftliche und politische Stärke zu gewinnen. Da die Ursachen von Extremismus vielfältig sind, bedarf es zur Prävention und Bekämpfung eines gesamtgesellschaftlichen Ansatzes. Am effektivsten kann dies erfolgen, wenn sich alle lokalen gesellschaftlichen Akteure bierzu vernetzen. Dies betrifft neben Kommunalverwaltung und Polizei auch Schulen sowie sonstige Bildungseinrichtungen, kulturelle und sportliche Organisationen und Verbände, Beratungsstellen, Ausländerbeiräte, Kirchen, Gewerkschaften, Jugendverbände, Schülerräte, Wirtschaftsorganisationen, aber auch die Bürgerinnen und Bürger selbst. Darüber hinaus müssen die Städte und Gemeinden sowohl von Bund und Ländern als auch von allen demokratischen Parteien und Organisationen Unterstützung erhalten.

In den letzten Jahren sind zunehmend Taten rechtsextremer Gruppierungen in den Blick der Öffentlichkeit geraten. Der jährliche Verfassungsschutzbericht des Bundesinnenministeriums und des Bundesamtes für Verfassungsschutz bezeugt eine im Wesentlichen gleich bleibend hohe Zahl von Organisationen und Personenzusammenschlüssen mit rechtsoder linksextremistischen Hintergrund. Die Anzahl rechtsextrem motivierter Straftaten erreichte 2008 ihren bisherigen Höhepunkt. Es wurden rund 18.000 rechtsextreme Straftaten, davon 965 Gewaltstraftaten, verübt. In Teilen Ost- wie Westdeutschlands wächst die Zustimmung zu ausländerfeindlichen und rechtsextremen Aussagen ebenso wie die Unzufriedenheit mit demokratischen System. Diese vorhandene Staatsverdrossenheit wird von rechtsextremen Gruppierungen ausgenutzt, wenn diese versuchen, mit verschiedenen Strategien gesellschaftspolitische Akzeptanz und politische Stärke zu gewinnen. Die Perspektiv- und Orien- tierungslosigkeit Einzelner wird für eigene Zwecke genutzt.

\section{Rechtsextremismus in Kommunen}

Viele Städte und Gemeinden in Deutschland erleben derzeit gezielte Aktivitäten rechtsextremistischer Gruppierungen und Parteien. Dabei handelt es sich nicht nur um Versammlungen, Demonstrationen oder Aufmärsche an bestimmten Orten und Plätzen oder für die Rechtsextremen bedeutsamen Tage. Auch der Kauf von Immobilien wird zunehmend zu einen] kommunalpolitischen Thema mit überregionaler Aufmerksamkeit.

Fast noch bedeutungsvoller ist, dass Rechtsextreme versuchen, in der Mitte der kommunalen Gesellschaft anzukommen. Neben der parlamentarischen Arbeit, dort wo sie in die Gemeindevertretungen gewählt werden, geschieht dies insbesondere durch außerparlamentarische Arbeit, zum Beispiel durch die Gründung oder Unterwanderung von Bürgerinitiativen, durch den strategischen Aufbau von Kandidatinnen und Kandidaten für Bürgermeisterwahlen, aber auch durch eine breite Öffentlichkeitsarbeit, angefangen durch gezielte Werbung im Umfeld von Schulen, zum Beispiel durch die Verteilung von CDs, Musikkonzerte sowie den Verkauf von Produkten über den Versandhandel und in Läden, durch kulturelle Arbeit, Angebote des Sports bis hin zum Engagement in der Jugend- und Sozialarbeit. Dazu zählt die Beratung von Hartz IV-Empfängern, von Rentnern bis hin zu Jugendangeboten zum Beispiel als »nationale Jugendarbeit « in Jugendzentren. Damit wandelt sich das Auftreten der Rechtsextremen von offen aggressiven zu kommunalen Alltagsaktivitäten. Dies fällt immer dort leicht, wo die bürgerlichen Parteien kaum noch eine aktive Basis haben oder in den Kommunen nicht zuletzt 
aufgrund der Finanzsituation keine Mittel für die Jugend und Kulturarbeit, die Unterstützung von Vereinen und Verbänden, zur Verfügung stehen.

Gleichwohl muss den sehr gern öffentlichkeitswirksam geäußerten Vorwürfen entgegengetreten werden, die Kommunen engagierten sich nicht gegen Rechts. Es mag immer negative Beispiele geben, richtig ist aber, dass die Städte und Gemeinden sich seit Jahren an den Aktivitäten zur Bekämpfung des Rechtsextremismus beteiligen. Kommunale Beiträge liegen beispielsweise in:

- der qualitätsorientierten Weiterentwicklung von Angeboten für Kinder und Jugendliche

- der Unterstützung von Vereinen und Verbänden des Sports und der Kultur

- der Unterstützung zivilgesellschaftlicher Kooperationen gegen Rechtsextremismus, Ausländerfeindlichkeit und Antisemitismus

- Aufbau vernetzter Präventionsarbeit, unter anderem im Rahmen von kriminalpräventiven »Runden Tischen «

- der Kooperation mit den Schulen sowie in der Bildungsarbeit kommunaler Volksschulen

- kommunalen Aktivitäten zur Integration von Ausländern und Aussiedlern

- der konsequenten Anwendung des rechtlichen Instrumentariums.

Diese Bandbreite von Maßnahmen zeigt, dass der notwendige Umgang mit den aktuellen Aktivitäten rechtsextremistischer Gruppierungen eine Vielzahl von Maßnahmen auf kommunaler Ebene bedingt. Soweit Kritik von Bundes- und Landespolitik an der angeblichen Untätigkeit der Kommunen laut wird, sind gerade diese Ebenen aufgerufen, die Kommunen nicht im Stich zu lassen und ihre Möglichkeiten, zum Beispiel durch Schärfung des rechtlichen Handlungsrahmens oder dadurch auszuschöpfen, dass Städte und Gemeinden über die notwendigen finanziellen Handlungsmöglichkeiten verfügen.

\section{Rechtsrahmen ausschöpfen}

Die Kommunen machen von den Vorschriften des Versammlungsgesetzes Gebrauch und unterstützen die Polizei und die Justiz bei ihren inzwischen erweiterten Eingriffsmöglichkeiten. Seit 2005 kann eine Versammlung oder ein Aufzug ver- boten oder von bestimmten Auflagen abhängig gemacht werden, wenn die Versammlung oder der Aufzug an einem Ort stattfindet, der als Gedenkstätte von historisch herausragender, überregionaler Bedeutung an die Opfer der menschenunwürdigen Behandlung und der nationalsozialistischen Gewalt- und Willkürherrschaft erinnert und nach den zur Zeit des Erlasses der Verfügung konkret feststellbaren Umständen zu befürchten ist, dass durch die Versammlung oder den Aufzug die Würde der Opfer beeinträchtigt wird.
Versammlungsrechts unterhalb der Schwelle eines Versammlungsverbotes aus. Die öffentliche Ordnung kann betroffen sein, wenn an einem bestimmten Tag ein in der Gesellschaft eindeutiger Sinngehalt mit gewichtiger Symbolkraft zukommt, der bei Durchführung eines Aufzugs an diesem Tag in dieser Weise angegriffen wird, dass dadurch zugleich grundlegende soziale oder ethische Anschauungen in erheblicher Weise verletzt werden. « (BVerfG vom 26.1.2001 1 $1 \mathrm{BvQ}$ 9/01) »So sind Einschränkungen

\section{"Rechtsextreme Gruppierungen haben immer dann Erfolg, wenn sie sich in den sozialen und politischen Strukturen vor Ort verankern können"}

Ebenfalls seit 2005 wurde in $\mathbb{S} 130$ Strafgesetzbuch ein neuer Absatz 4 eingefügt, demzufolge »mit Freiheitsstrafe bis zu drei Jahren oder mit Geldstrafe bestraft wird, wer öffentlich oder in einer Versammlung den öffentlichen Frieden in einer die Würde der Opfer verletzenden Weise dadurch stört, dass er die nationalsozialistische Gewalt- und Willkürherrschaft billigt, verherrlicht oder rechtfertigt «.

Dass diese Möglichkeiten oft nur ein stumpfes Schwert bleiben, zeigt der Fall der Stadt Jena: Die Stadt erließ ein Versammlungsverbot, wohl wissend, dass die Begründung eher dürftig war. Die Verfügung wurde vom Gericht aber nicht nur aufgehoben, sondern es bescheinigte dem Oberbürgermeister, dass mit seiner Verfügung der Straftatbestand der Rechtsbeugung »erfüllt sein kann«.

Mit Blick auf das Versammlungsrecht ist auf die sogenannte Auflagenrechtsprechung des Bundesverfassungsgerichts hinzuweisen. Es lässt unter dem Gesichtspunkt des Schutzes der öffentlichen Ordnung Korrekturen der Gestaltung einer Versammlung zu, wobei folgende Einschränkungsmöglichkeiten zum Tragen kommen: "Die öffentliche Ordnung scheidet jedenfalls nicht grundsätzlich als Schutzgut für eine Einschränkung des der Versammlungsfreiheit verfassungsrechtlich unbedenklich, die ein aggressives und provokatives, die Bürger einschüchterndes Verhalten der Versammlungsteilnehmer verhindern sollen, durch das ein Klima der Gewaltdemonstration und potenzieller Gewaltbereitschaft erzeugt wird. Die öffentliche Ordnung kann auch verletzt sein, wenn Rechtsextremisten einen Aufzug in einem speziell der Erinnerung an das Unrecht des $\mathrm{Na}-$ tionalsozialismus und den Holocaust dienenden Feiertag so durchführen, dass von seiner Art und Weise Provokationen ausgehen, die das sittliche Empfinden der Bürgerinnen und Bürger erheblich beeinträchtigen.« (BVerfG vom 23.6.2004 1BvQ 19/04)

Nicht verschwiegen werden soll allerdings folgende praktische Problematik: Vielfach engagieren sich die Städte mit ihren Bürgerinnen und Bürgern, Vereinen und Verbänden in Gegendemonstrationen gegen rechtsextremistische Versammlungen. Aufgrund des verfassungsrechtlichen Schutzes der Versammlungsfreiheit werden diese Gegendemonstrationen untersagt, soweit der Aufmarsch rechtsextremistischer Gruppierungen nach den genannten Grundsätzen nicht verboten werden kann. In diesen Fällen muss Verständnis für die Polizei aufgebracht wer- 
den, die rechtlich gezwungen ist, auch rechtsextremistische Versammlungen zu beschützen. Leider ist $\mathrm{zu}$ beobachten, dass bei Gegendemonstrationen immer wieder gewaltbereite Demonstranten den Einsatz von Polizisten provozieren mit der Folge, dass entweder negativ über den Polizeieinsatz berichtet oder die gesamte Veranstaltung der rechtsextremen Seite pressemäßig »aufgewertet « wird. Dies passiert selbst dann, wenn nach einer großen Ankündigung nur eine Handvoll rechtsextremistischer Demonstrationsteilnehmer erschienen sind.

Hinsichtlich der Nutzung kommunaler Einrichtungen ist auf eine bemerkenswerte Entscheidung des Niedersächsischen Oberverwaltungsgerichts (10 OB 231/07 vom 24.10.2007) hinzuweisen. Das Gericht ließ erstinstanzliche Entscheidungen unbeanstandet, wonach eine von der Kommune in Form der GmbH betriebene Stadthalle nicht an die NPD verpachtet wurde. So führt das VG Oldenburg (1 B 2488) aus, dass die GmbH in ihrer Entscheidung autonom sei. Die Überlassung der Räume auch an politische Parteien sei der $\mathrm{GmbH}$, insbesondere ihrer Geschäftsführung, zur alleinigen Entscheidung überlassen. Einflussnahmen der Stadt, etwa zur Verwirklichung von Ansprüchen aus der Gemeindeordnung oder dem Parteiengesetz, seien nicht vorgesehen und werden auch nicht wahrgenommen. $\mathrm{Ob}$ und inwieweit die Antragstellerin Ansprüche gegen die $\mathrm{GmbH}$ auf Abschluss eines Pachtvertrages habe, sei nicht zu entscheiden. Dies wäre ein ausschließlich zivilrechtlicher Anspruch, der vor dem zuständigen ordentlichen Gericht $\mathrm{zu}$ verfolgen wäre. Einen solchen Kontrahierungszwang hat das Landgericht Oldenburg (3 0 2862/07) aber ebenfalls abgelehnt.

Ein weiteres problematisches Themenfeld sind Immobilienkäufe. Das Problem besteht dabei darin, zum einen zu erkennen, ob rechtsextreme Käufer auftreten. Anhaltspunkte können Kontakte des Verkäufers zum rechtsextremen Spektrum sein, eine große Diskrepanz zwischen der Verkaufssumme und dem Verkaufswert, die Einschaltung eines Maklers oder eines Beauftragten, der eine hohe Provision verlangt, oder eine mediale Vermarktung des lmmobilienkaufes. In Zweifelsfällen sollten sich die Städte und Gemeinden an übergeordnete Dienststellen, den Verfassungsschutz oder das zuständige Ministe- rium wenden, um Informationen über die Hintergründe des Käufers zu erhalten. Aus der Praxis werden im Übrigen folgende rechtliche Instrumente genannt, Verkäufe zu verhindern: die Ausübung des städtischen Vorkaufsrechtes, die Erweiterung des Sanierungsgebietes sowie Möglichkeiten nach dem Denkmalschutz oder dem Brandschutzgesetz.

\section{Infrastruktur stärken}

Neben den rechtlichen Instrumenten sind die Kommunen insbesondere gefordert, eine Infrastruktur vorzuhalten, die es den rechtsextremistischen Gruppierungen nicht erlaubt, etwa durch eigene "Jugendarbeit «Fuß zu fassen. So sind die Städte zum Beispiel in den Aufgabenfeldern Jugendarbeit, außerschulische Bildung, Migration und Unterstützung von Sportvereinen gefordert. Besonders zu erwähnen sind neue Angebote der offenen Jugendarbeit, die Schaffung von Freizeitangeboten sowie die Förderung des Vereinslebens.

Dabei darf nicht übersehen werden, dass nicht nur Jugendliche empfänglich gegenüber rechtsextremen Gedankengutes sind. Der Erfolg rechtsextremer Initiativen, Gruppierungen und Parteien hängt immer damit zusammen, dass es ihnen zunehmend gelingt, sich in den sozialen und politischen Strukturen vor Ort $\mathrm{zu}$ verankern. Diesen Entwicklungen ist durch eine Stärkung zivilgesellschaftlicher Strukturen und eine klare Abgrenzung von Akteuren mit einem rechtsextremistischen Hintergrund $\mathrm{zu}$ begegnen. Die Unterstützung des Vereinslebens vor Ort, nicht nur im Sport, sollte dazu genutzt werden, allen Einwohnerinnen und Einwohnern, egal welchen Alters, mit oder ohne Migrationshintergrund, Angebote zu machen, damit sie nicht zu Opfern gezielter Angebote rechtsextremer Organisationen werden.

\section{Kommunen brauchen Unterstützung}

Im Rahmen ihrer Möglichkeiten sind die Kommunen bereit, ihren Anteil an den gemeinsamen Anstrengungen aller Beteiligten $\mathrm{zu}$ leisten. Die verfassungsrechtliche und die finanzielle Situation der Kommunen setzt hier allerdings auch
Grenzen. Gesamtgesellschaftliche Entwicklungen müssen von allen Ebenen des Staates sowie von den zivilgesellschaftlichen Akteuren insgesamt angegangen werden. So sind die Länder gefordert. die Polizeipräsenz auch in der Fläche aufrecht zu erhalten. Bund und Länder müssen die Förderung ländlicher Räume wieder stärker in den Blick nehmen. Modellprogramme sind ein Ansatz, helfen aber nur einzelnen Kommunen und sind vielfach nicht nachhaltig genug.

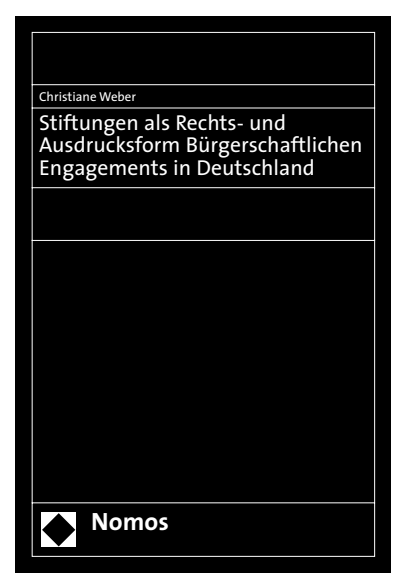

Stiftungen als Rechts- und Ausdrucksform Bürgerschaftlichen Engagements in Deutschland

Von Christiane Weber 2009, 239 S., brosch., 54,- $€$, ISBN 978-3-8329-3866-6

Das Buch eröffnet einen kritischen Zugang zum sozialen, historischen und rechtlichen Phänomen der Stiftung und stellt den Zusammenhang mit dem Begriff des Bürgerschaftlichen Engagements sowie dem aktuellen Gemeinnützigkeitsrecht her. Eine Bestandsaufnahme gibt Einblick in die Vielfalt des Stiftungswesens und die Beweggründe, eine Stiftung ins Leben zu rufen.

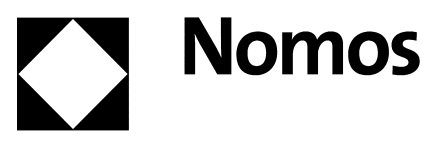

Bitte bestellen Sie im Buchhandel oder versandkostenfrei unter $\downarrow$ www.nomos-shop.de 